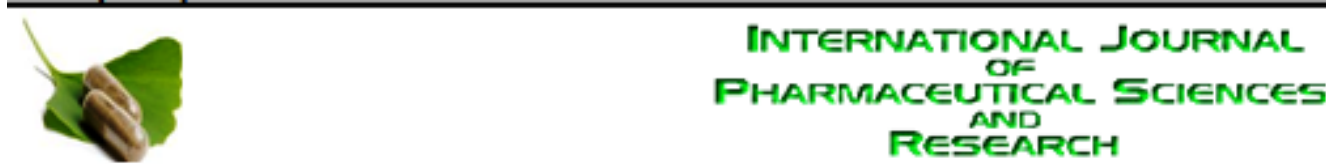

Received on 15 September, 2012; received in revised form, 15 November, 2012; accepted, 17 December, 2012

\title{
SONOPHORESIS EFFECT ON THE PERMEATION OF METRONIDAZOLE USING 3D SKIN EQUIVALENT
}

\section{Mai Aldwaikat*1, ${ }^{2}$, Mohammed Alarjah ${ }^{2}$, Jacki Willis ${ }^{3}$, Timothy Mason ${ }^{1}$}

Sonochemistry Applied Research Centre, Coventry University ${ }^{1}$, Coventry, CV1 5FB, England

Faculty of Pharmacy, Umm Alqura University ${ }^{2}$, Makkah, KSA

School of Life Science, University of Hertfordshire ${ }^{3}$, Hertfordshire, AL10 9AB, England

\section{Keywords:}

Sonophoresis, Metronidazole, skin permeation, 3D skin equivalent

Correspondence to Author:

Mai Aldwaikat

Sonochemistry Applied Research Centre, Coventry University, Coventry, CV1 5FB, England

E-mail: maidwaik@yahoo.co.uk

\begin{tabular}{|c|c|}
\hline QUICK RESPONSE CODE & \\
\hline \multirow{2}{*}{ 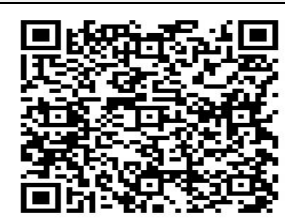 } & $\begin{array}{c}\text { IJPSR: } \\
\text { ICV- } 5.07\end{array}$ \\
\hline & $\begin{array}{c}\text { Website: } \\
\text { www.ijpsr.com }\end{array}$ \\
\hline
\end{tabular}

\begin{abstract}
Transdermal drug delivery is highly advantageous method for drug administration, yet the compact structure of stratum corneum is an effective barrier which limited the use of this route to very few drug molecules. Ultrasound enhances the permeation through the skin by altering this barrier function in a phenomenon named sonophoresis. This study aimed to evaluate the effect of variable ultrasonic conditions on the permeation of Metronidazole (ML). This involved validating the applicability of utilizing a three dimensional skin equivalent as human skin models for sonophoresis studies. A Franz diffusion cell was used to study the effect of therapeutic ultrasound at $1 \mathrm{MHz}$ and power ultrasound at $20 \mathrm{KHz}$ on the permeation of Metronidazole across the 3D skin equivalent EpiDerm ${ }^{\mathrm{TM}}$. HPLC methods were used for the determination of the concentration of Metronidazole in the receiving compartment. Utilizing therapeutic ultrasound at $1 \mathrm{MHz}$ frequency has enhanced the permeation of Metronidazole through the EpiDerm ${ }^{\mathrm{TM}}$, as about double the permeation of the ML was obtained with no apparent damage to the membrane. The results indicated significant permeation enhancement with low frequency sonophoresis at $20 \mathrm{KHz}$ frequency treatments through EpiDerm ${ }^{\mathrm{TM}}$ skin equivalent. Up to five fold enhancement was measured with the permeation of investigated molecules through EpiDerm $^{\mathrm{TM}}$. The mechanism of action observed seemed highly dependent on the ultrasonic conditions.
\end{abstract}

INTRODUCTION: Metronidazole is a nitroimidazole anti-infective drug used in the treatment of the dermatological condition called rosacea (adult acne). It is produced in a gel form of $0.75 \%$ for twice daily application and recently in $1 \%$ strength for once a day application. As transdermal drug delivery has many advantages over oral administration delivery route ${ }^{1,2}$. The advantages of transdermal drug delivery have made the research efforts to overcome the major element of resistance, the stratum corneum SC, viable and worthwhile.
Many researches are currently work in alteration the barrier function of stratum corneum that limits the conventional use of the skin as an administrative route ${ }^{3}$. SEPA (Soft Enhancement of Percutaneous Absorption) agent which is considered as chemical enhancer is used to increase skin permeability without causing irritation 4, 5 . Iontrophoresis; which enhance the medicament penetration through the skin barrier by using low electrical potential gradient. Iontophoresis is mainly applicable to small molecules up to a few thousand Daltons ${ }^{5}$. 
Microneedles have been used to deliver solutions across the skin without causing pain because they do not reach the nerve endings ${ }^{1}$. The main drawback of the microneedle is the difficulties of its quality control and its low tolerance to the handling during manufacturing and use. Sonophoresis, which involves the application of an ultrasound (US) waveform to alter the stratum corneum SC barrier function, thus enhancing transdermal transport of drugs through skin by increasing percutaneous absorption of these drugs 6

Sonophoresis has recently been used in the medical field to improve transdermal drug transport and to achieve a more suitable therapeutic approach. With this technique the ultrasound is used to move molecular drugs through intact living skin and into the soft tissue 7. It is one of the most promising novel drug delivery system and has been shown to enhance the skin penetration and release rate of number of drugs that have poor absorption or permeation profiles through the skin.

It is convenient and rapid method of delivering low molecular weight drug as well as macromolecules into skin. Many articles have summarized various aspects relating to sonophoresis; overviewed application, mechanisms, factors that influence sonophoretic drug delivery, clinical studies, synergistic effect of ultrasound with other enhancement such as chemical enhancers and iontophoresis and the biological effects of ultrasound. This technique is painless and functions independently of the drug electrical characteristics ${ }^{8}$.

Ultrasound could be classified into low frequency ultrasound $(20-100 \mathrm{kHz})$ and therapeutic frequency ultrasound (1-3 $\mathrm{MHz}$ ). Most studies and clinical approach have been used low frequency ultrasound 9-11. Low frequency ultrasound has been widely used in transdermal drug delivery as it has been found to be more potent in enhancing skin permeability than therapeutic, which could be attributed to cavitations phenomena. Cavitation (the formation and collapse of gaseous cavities) has been suggested to be the main factor in the ability of low frequency ultrasound to increase the skin permeability. Taking this assumption into consideration can lead to a meaningful explanation of the phenomenon of sonophoresis 12. However; therapeutic ultrasound can enhance the transdermal permeation of low weight drugs.

In this study Metronidazole is used as drug model to study the effect of ultrasonic mechanical waves on the reversible alteration of skin natural barrier function. This will involve an investigation of the effect of different ultrasonic parameters: frequency, intensity and exposure time on skin permeability. Up to authors knowledge there is no information on sonophoretic studies of Metronidazole.

\section{MATERIALS AND METHODS:}

Materials: All chemicals used were of analytical grade and supplied by PDH -UK and used without extra purification. Metronidazole was pharmaceutical grade supplied by Degraaf pharmaceuticals. EpiDerm ${ }^{\circ}$ skin culture: EPI 200X from Mattek Corporation USA.

Metronidazole formulation: Metronidazole gel was prepared in a $0.75 \%$ concentration as follows: Metronidazole equivalent to 0.75 gram was dissolved in $50 \mathrm{~mL}$ of equal mixture of water absolute alcohol and propylene glycol. 7.5 gram of Sepigel- $305^{\mathrm{TM}}$ (Polyacrylamide/C13-14 Isoparaffin/laureth-7) was mixed with $50 \mathrm{~mL}$ of purified water with continuous stirring until the gel structure has formed. The metronidazole solution was then added to the formed gel with continuous mixing until a homogenous gel was obtained after adjusting the weight with purified water to $100 \mathrm{~g}$, the $\mathrm{pH}$ then was tested for $10 \%$ diluted gel and it was 7.2. The gel was left for 48 hours to settle prior to use. 1 gram of the prepared gel was used in each experiment.

Skin preparation: EpiDerm was utilized within three days from the receiving day, during which was kept refrigerated at $4-8{ }^{\circ} \mathrm{C}$. The tested patches were removed from the skin culture under vertical laminar flow to insure sterility and viability of the skin patches. The membranes were soaked in PBS $\mathrm{pH} 7.4$ for one hour before starting the permeation experiment. 


\section{Ultrasound System:}

1. Low Frequency Probe Ultrasound (20 KHz): The Sonics and Materials $20 \mathrm{KHz}$ probe model CV26 with a probe tip surface area of $1.77 \mathrm{~cm}^{2}$ was used in this research. This system was used either directly, by placing the top of the probe in the gel over the membrane in the donor compartment of the cell, or indirectly by exposing the membrane to the sonication irradiation after placing the membrane in a waterbath. The membrane would then be clamped in the cell to conduct the permeability test.

2. Physiotherapy Ultrasound Device: The Medi-Link system, dual frequency, Model No. 72, supplied by Electro-medical supplies (Greenham) was used for the conduction of this work. The device was composed of an electrical generator source and two probe transducers with a tip area of $0.5 \mathrm{~cm}^{2}$ and $5 \mathrm{~cm}^{2}$. The frequency could be switched from $1 \mathrm{MHz}$ to $3 \mathrm{MHz}$ and the intensity was adjustable from 0 to $2 \mathrm{~W} / \mathrm{cm}^{2}$. The $0.5 \mathrm{~cm}^{2}$ probe was used in the permeability enhancement tests, as it was possible to place it at the top of the membrane using the gel preparations as a propagating medium.

Determination of Ultrasound Intensity: The Intensity is defined as: the amounts of energy (power) crossing unit are in unit time. Intensity of ultrasound was determined using a calorimetric method. Water was placed in beaker and ultrasound was on for certain period of time (prefer for $1 \mathrm{~min}$.). The temperature of water was measured using thermometer during ultrasound activation. The intensity of ultrasound was calculated according to the following formula:

$$
I=\left(\frac{m \times C p}{A}\right) \frac{d T}{d t}
$$

Where $I$ is the intensity of ultrasound $\left(W / \mathrm{cm}^{2}\right), m$ is the mass of water, $C p$ is the specific heat capacity of water $\left(4.18 \mathrm{~J} / \mathrm{g}{ }^{\circ} \mathrm{C}\right), A$ is the surface area of the sonicator horn and $d T / d t$ is the rate of change of temperature of water.

\section{Optimization of Ultrasound parameters for} Transdermal and Topical Delivery of DS: For the performance of the sonication experiments, the EpiDerm $^{\mathrm{TM}}$ tissue was sonicated with the physiotherapeutic probe $\left(0.5 \mathrm{~cm}^{2}\right)$ using the medicated gel as a conducting medium. For the power sonication, the tissue was placed in $20 \mathrm{ml}$ PBS, a $1 \mathrm{~mm}$ thickness PTFE disc was placed at the bottom of the beaker to prevent US beam reflections. The tissue was irradiated for the specified time with the probe, which was adjusted to be 1 or $2 \mathrm{~cm}$ away from the surface of the tissue. The section was checked visually for the intactness, prior to being fixed at the top of the Franz cell, using $0.2 \%$ sodium fluorescein (SF) solution in the donor compartment.

SF is a large, highly polar molecule, unable to penetrate the intact skin structure and its penetration tested using a spectrofluorometer with the excitation and emission wavelengths set at 488 and $510 \mathrm{~nm}$, respectively. For delivery experimental 1 gram of the ML gel preparation was placed in the donor compartment at the top of the EpiDerm ${ }^{\circledR}$ insert previously washed with PBS .The receiving compartment was filled with PBS $\mathrm{pH}$ 7.4: propylene glycol (70:30) and stirred constantly at $700 \mathrm{rpm}$ with helix stirrer and magnetic bar. The effect of physiotherapeutic $1 \mathrm{MHz}$ ultrasound and the power $20 \mathrm{KHz}$ ultrasound, on the permeation of $\mathrm{ML}$ through EpiDerm $^{\mathrm{TM}}$ skin culture was studied, at 10 and 20 minute irradiation time intervals for $1 \mathrm{MHz}$ frequency and $10 \%, 15 \%$ and $20 \%$ amplitudes for the $20 \mathrm{KHz}$ frequency with different parameters and mode of sonication see table.

Diffusion Cell set up: The permeation experiments were conducted with a vertical Franz diffusion cell with a $12.0 \mathrm{~mL}$ volume receptor compartment and $1.77 \mathrm{~cm}^{2}$ diffusion area, supplied by Hanson USA. It was used for the evaluation of the effect of different ultrasonic parameters on the permeability of metronidazole. For the study of the permeation through skin culture, $1 \mathrm{~mm}$ thickness PTFE part with an orifice of a $0.5 \mathrm{~cm}$ was fixed on the top of the cell, between the donor and the receiving compartments as the diameter of the skin culture was $0.8 \mathrm{~cm}$. the cell temperature was maintained at $32 \pm 2{ }^{\circ} \mathrm{C}$ using the water jacket system surrounding the cell and circulating water bath. Samples of $0.5 \mathrm{~mL}$, at 1-hour intervals were removed using a microlitre syringe, with immediate replacement of the sample with an equal volume of fresh receptor medium. 
Metronidazole HPLC Determination: The standard BP analytical method (BP, 1998) with slight modification was used for the determination of Metronidazole using Waters Alliance HPLC (Waters Corp, Milford, MA, USA) equipped waters e2995 separation module and waters 2998 PDA and Empower 2 software. The mobile phase was an isocratic system prepared by mixing methanol and $0.1 \mathrm{M}$ monobasic phosphate buffer in a ratio of (70:30). Column: Stainless steel, reverse phase, $C_{18}$ column, $5 \mu \mathrm{m}, 4.6 \mathrm{~mm} \times 150 \mathrm{~mm} \times$ Bridge. Detector: UV - at $314 \mathrm{~nm}$. Flow Rate: $1.0 \mathrm{~mL} / \mathrm{min}$. Sample volume (loop): $100 \mu \mathrm{L}$. Solvent phosphate buffer 0.1M. A very well resolved peak of metronidazole was obtained with a retention time of about 3.7 minutes. Calibration curves were prepared in 0.9 to $6 \mathrm{mg}$ per $100 \mathrm{~mL}$. The minimum detection limit and quantitation limit for $\mathrm{ML}$ using the above mentioned HPLC method was calculated.

Skin permeation (Calculation Aspects): Fick's laws can be applied for skin permeation; the final form of the equation after modification ${ }^{12}$ is as follow:

$$
J=\frac{\partial M}{\partial t}=\frac{D P C_{d}}{h}
$$

Where $J$ is the flux of a permeate through a barrier, $M$ is the cumulative amount of the permeant, $D$ is diffusion coefficient of the permeant, $h$ is the barrier thickness, $P$ is partition coefficient of the permeant between the vehicle and the barrier, and $C_{d}$ is concentration in the donor compartment. Permeability coefficient $K_{p}$ of the permeant through the barrier can be obtain from the following equation ${ }^{12}$ :

$$
K_{p}=\frac{J}{C_{d}}
$$

The enhancement ratios (ER) were calculated as following

$$
E R=\frac{J_{E}}{J_{p}} * 100 \%
$$

Where $J_{E}$ is enhanced flux obtained post the ultrasonic treatment of the barrier, and $J_{p}$ is passive flux obtained with control experiments. The amount of drug accumulated in the receiving compartment was calculated by using the equation of the line for the calibration curve of the drug:

$$
\left(\frac{A}{S}\right) * d *\left(\frac{12}{0.5}\right)+p
$$

Where $A$ is Analytical instrument signal i.e area under the curve of the sample at time $t$ (hrs), $S$ is the slope of calibration curve, $d$ is the dilution factor, 12 is the receiving chamber volume, 0.5 is sample volume, $P$ is the amount of drug in $\mathrm{mg}$ in the previous samples. A plot of the cumulative amount in $\mathrm{mg}$ versus time in hours in order to calculate pseudo state -state flux was performed.

Statistical evaluation: The ANOVA one-way analysis was used to evaluate the permeation of the drugs under investigation, between control experiments and those performed after US exposure, adopting a 5\% level of significance. Post hoc tests using the least significant difference between means were conducted to identify differences between treatments. The statistical analysis was conducted using SPSS 14 software package.

RESULTS: Transdermal delivery of different drugs molecules has been limited by the barrier properties of skin. Three main routes could be taken place when material across the skin:

1. The intracellular route.

2. The intercellular route.

3. A parallel passage, through the skin appendages.

This route has a limited role in transdermal penetration as these structures represent less than $1 \%$ of the total skin surface area ${ }^{13,14}$. The appendages structures are not present in EpiDerm ${ }^{\mathrm{TM}}$.

Transdermal permeation of drugs could be enhanced significantly by using Sonophoresis. This technique has been commonly used to assist delivery of topically applied anesthetics, counter irritants and antiinflammatory agents ${ }^{15}$. In this study ultrasound is used as enhancement technique, we have investigated the possibility of achieving significant permeation of skin using lower application times. The cumulative amounts of the drug together with the coefficient of variation were calculated for each condition. The pseudo-steady state flux was calculated from the gradient of the linear 
portion of the permeation profile for each individual profile. Experiment conditions used to investigate the effect of ultrasound on metronidazole permeation across Epiderm skin culture are summarized in Table 1.

A control experiment was conducted using the same procedures and parameters but without any ultrasound treatment. Effect of ultrasound parameters on the Metronidazole solution was investigated. The mean value of the recovered amount of $M L$ in a solution is shown in Table 2, after the exposure to different US conditions, versus the control solutions, it also shows the standard deviation and coefficient of variation. ANOVA one way analysis was performed to evaluate the significant of the difference between the control experiment and ultrasonic experiments.

At $\mathrm{P}<0.05$ there was no significant difference. Table 3 gives a summary of all ultrasonic experiments performed to study the permeation of ML across the EpiDerm $^{\text {TM }}$ barrier versus the control. It shows the US conditions and number of trials performed for each investigation, the mean flux ( $\pm 1 \mathrm{SD}$ ), the permeability coefficient and percentage enhancement of the drug compared with control. The energy density of the power ultrasonic conditions is also presented.

TABLE 1: SUMMARY OF EXPERIMENTAL CONDITIONS STUDYING THE EFFECT OF US ON ML PERMEATION ACROSS EPIDERm ${ }^{\text {TM }}$

\begin{tabular}{|c|c|c|c|c|c|}
\hline Test & US frequency & Amplitude & Mode & Sonication time (min) & Sonication condition \\
\hline Control & NA & NA & NA & NA & NA \\
\hline Physiotherapy US & $1 \mathrm{MHz}$ & NA & Continuous & 10 & $\begin{array}{l}\text { US probe placed } 1 \mathrm{~cm} \text { away from the } \\
\text { membrane }\end{array}$ \\
\hline Physiotherapy US & $1 \mathrm{MHz}$ & NA & continuous & 20 & $\begin{array}{l}\text { US probe placed } 1 \mathrm{~cm} \text { away from the } \\
\text { membrane }\end{array}$ \\
\hline Low frequency & $20 \mathrm{KHz}$ & $10 \%$ & Continuous & 3 & $\begin{array}{l}\text { US probe placed } 2 \mathrm{~cm} \text { away from the } \\
\text { membrane }\end{array}$ \\
\hline Low frequency & $20 \mathrm{KHz}$ & $10 \%$ & 1:1 Pulsed & 5 & $\begin{array}{c}\text { US probe placed } 2 \mathrm{~cm} \text { away from the } \\
\text { membrane }\end{array}$ \\
\hline Low frequency & $20 \mathrm{KHz}$ & $10 \%$ & Continuous & 5 & $\begin{array}{l}\text { US probe placed } 2 \mathrm{~cm} \text { away from the } \\
\text { membrane }\end{array}$ \\
\hline Low frequency & $20 \mathrm{KHz}$ & $15 \%$ & Continuous & 5 & $\begin{array}{l}\text { US probe placed } 2 \mathrm{~cm} \text { away from the } \\
\text { membrane }\end{array}$ \\
\hline Low frequency & $20 \mathrm{KHz}$ & $20 \%$ & Continuous & 5 & $\begin{array}{c}\text { US probe placed } 2 \mathrm{~cm} \text { away from the } \\
\text { membrane }\end{array}$ \\
\hline
\end{tabular}

TABLE 2: RECOVERED AMOUNT OF ML (MG/100ML) FOLLOWING THE EXPOSURE TO DIFFERENT US CONDITION VERSUS CONTROL

\begin{tabular}{cccc}
\hline & Control & 20KHz, 20\%, 20min & 1MHz 20 min \\
\hline $\mathbf{n}$ & 6 & 6 & 6 \\
Mean & 0.9912 & 0.9883 & 0.9871 \\
SD & 0.009 & 0.007 & 0.004 \\
COV\% & 0.91 & 0.71 & 0.41 \\
\hline
\end{tabular}

TABLE 3: SUMMARY OF ULTRASONIC PARAMETERS USED, AND THE FLUX FINDINGS OF ML ACROSS EPIDERm ${ }^{\circledR}$ SKIN CULTURE, UNDER DIFFERENT US CONDITIONS

\begin{tabular}{|c|c|c|c|c|c|c|c|c|c|c|c|}
\hline US Parameter & $\mathbf{F}$ & Amp. \% & I & $\mathrm{W} / \mathrm{cm}^{2}$ & $\begin{array}{l}\text { US time } \\
\text { (min) }\end{array}$ & mode & $\mathbf{n}$ & $\begin{array}{c}\text { Flux mean } \pm \text { SD } \\
\mathrm{mg} / \mathrm{cm}^{2} \cdot \mathrm{hr}\end{array}$ & $\begin{array}{c}\mathrm{Kp} \\
\mathrm{cm} / \mathrm{hr}\end{array}$ & ED & Enh\% \\
\hline Control & - & - & & - & - & - & 5 & $0.1056 \pm 0.029$ & 0.014 & 0 & 100 \\
\hline Physiotherapy US & $1 \mathrm{MHz}$ & - & & 2 & 10 & Continuous & 3 & $0.1724 \pm 0.029$ & 0.022 & - & 163 \\
\hline Physiotherapy US & $1 \mathrm{MHz}$ & - & & 2 & 20 & Continuous & 4 & $0.2091 \pm 0.199$ & 0.028 & - & 198 \\
\hline Low frequency US & $20 \mathrm{KHz}$ & $10 \%$ & & 2.85 & 3 & Continuous & 3 & $0.1052 \pm 0.025$ & 0.014 & 504 & 99.6 \\
\hline Low frequency US & $20 \mathrm{KHz}$ & $10 \%$ & & 1.87 & 5 & pulsed & 3 & $0.1098 \pm 0.063$ & 0.015 & 480 & 104 \\
\hline Low frequency US & $20 \mathrm{KHz}$ & $10 \%$ & & 2.85 & 5 & Continuous & 5 & $0.1688 \pm 0.039$ & 0.023 & 855 & 160 \\
\hline Low frequency US & $20 \mathrm{KHz}$ & $15 \%$ & & 5.24 & 5 & Continuous & 3 & $0.499 \pm 0.25$ & 0.067 & 1572 & 473 \\
\hline Low frequency US & $20 \mathrm{KHz}$ & $20 \%$ & & 7.02 & 5 & Continuous & 5 & $0.468 \pm 0.278$ & 0.0632 & 2106 & 443 \\
\hline
\end{tabular}

F: frequency, I: Intensity US: ultrasound, Enh: Enhancement, Amp: Amplitude, Kp: permeability coefficient ED: energy density. 


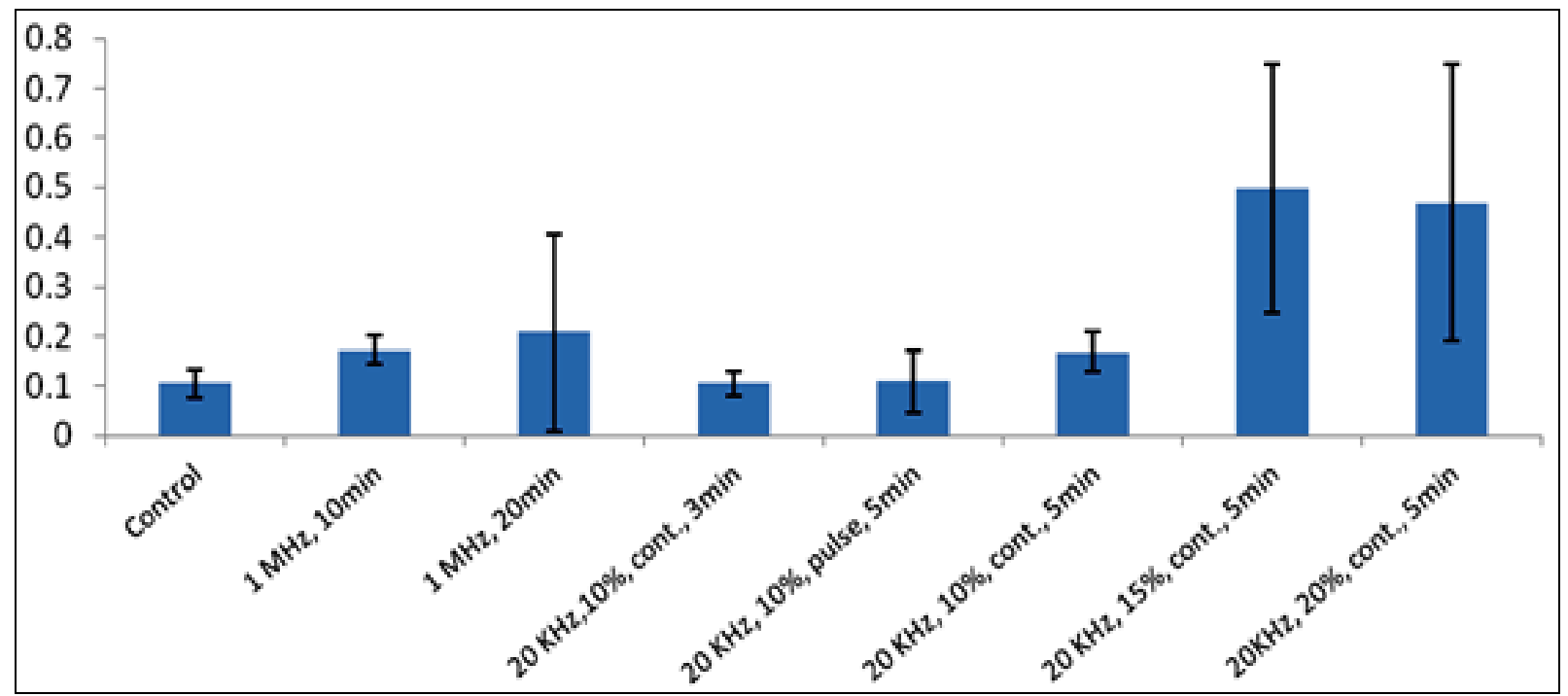

FIGURE 1: ML FLUX THROUGH EpiDerm ${ }^{\text {TM }}$ VERSUS ULTRASOUND CONDITIONS. (Mean $\pm 1 S D$, (n minimum 3)). ${ }^{*} \mathrm{P}<0.05$

Physiotherapeutic US: Therapeutic sonophoresis was used to test their ability to enhance the permeability through the skin. Although physiotherapeutic application of ultrasound utilizes a frequency range from 1 to $3 \mathrm{MHz}, 1 \mathrm{MHz}$ was chosen as it was reported that the enhancement ratio is inversely proportion to the frequency used in the treatment. Ultrasound at 1 $\mathrm{MHz}$ frequency $2 \mathrm{~W} / \mathrm{cm}^{2}$ intensity and continuous mode for 10 minutes gave $163 \%$, the sonication was increased to 20 minutes to check the effect of time and the enhancement was found to be $198 \%$. None of these enhancements were significant compared to the control.

These results are compatible with other research work where the sonophoretic treatments using $1 \mathrm{MHz}$ show no enhancement or a moderate improvement. The discrepancies in the level of the penetration enhancement obtained utilizing therapeutic ultrasound could be due to the variations of physicochemical properties of the investigated molecules and their vehicles ${ }^{16,17}$.

Figure 2 demonstrates the effect of three different exposure time of physiotherapeutic sonication at 1 $\mathrm{MHz}$ directly on the permeation of $\mathrm{ML}$ through EpiDerm $^{\text {TM }}$ skin section compared with the control treatment. Thermal effect of sonophorsis and reversibility of US effect on skin should be considered. In our case any thermal effect could be excluded from being the main enhancing cause as the irradiation was carried out in the BPS buffer prior to the permeation test and by the time of fixing the tissue in a Franz cell, the tissue is at room temperature and then its temperature was increased to $32^{\circ} \mathrm{C}$ which is the temperature of the set-up.

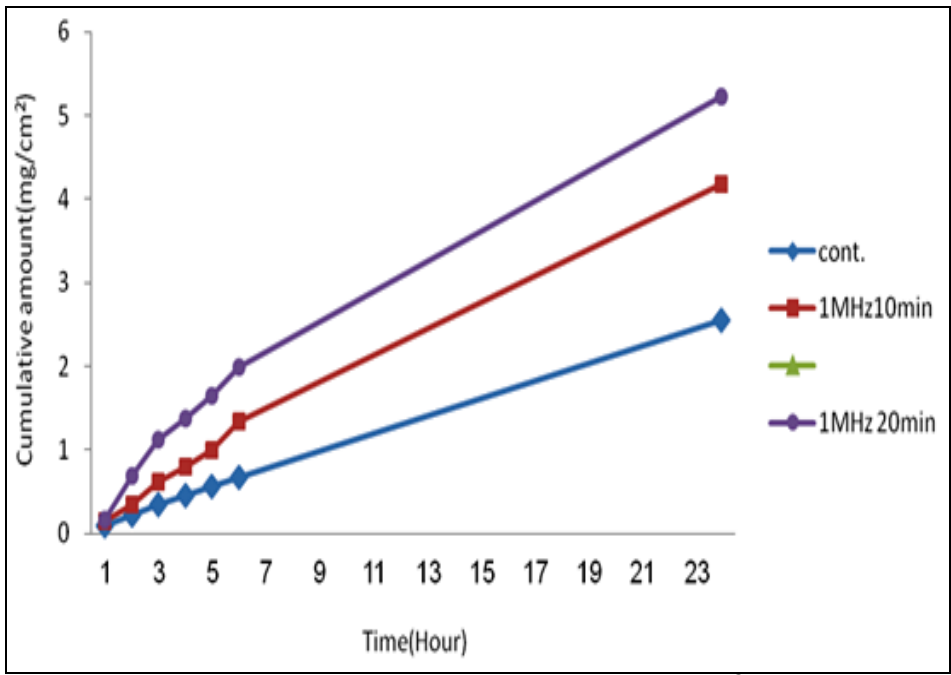

FIGURE 2: EFFECT OF $1 \mathrm{MHz}$ SONICATION, $2 \mathrm{~W} / \mathrm{cm}^{2}$ FOR $10 \mathrm{MIN}$, 15 MIN AND 20 MIN CONTINUOUSLY ON ML PERMEATION THROUGH EpiDerm ${ }^{\text {TM }}$.

Power US: Low frequency sonophoresis of EpiDerm ${ }^{\text {TM }}$ skin equivalent showed distinctive permeation enhancement for the investigated molecule. Table 3 demonstrates the effect of 3 minutes of continuous sonication at $20 \mathrm{KHz} 10 \%$ amplitude, 5 minutes of 20 $\mathrm{KHz}$ 10\% 0.1:0.1 pulsed mode, 5 minutes continuous sonication at $20 \mathrm{KHz} 10 \%$ amplitude, 5 minutes continuous sonication at $20 \mathrm{KHz} 15 \%$ amplitude and 5 minutes continuous sonication at $20 \mathrm{KHz} \quad 20 \%$ amplitude on the permeation of ML across EpiDerm ${ }^{\text {TM }}$. $99.6 \%, 104 \%, 160 \%, 473 \%$, and $443 \%$ enhancement ratios were achieved respectively. 
The best results were obtained with $20 \mathrm{KHz}$ sonication for 5 minutes $15 \%$ and $20 \%$ intensity, the conflict in the result between $15 \%$ and $20 \%$ could be due to the large standard deviation found for most of the data points of cumulative amount-time profiles of the permeation across this tissue, however further alteration of the parameters by increasing the time of exposure in the case of $20 \mathrm{KHz}$ irradiation or the intensity or by decreasing the distance between the probe and the membrane during sonication was not possible due to the fragile nature of the membrane as visually detected defects were observed upon increasing the time or the intensity of irradiation.

This observation suggests the creation of tiny holes within the texture of the membrane, which increased the permeation of ML through skin. Figure $\mathbf{3}$ shows the effect of different amplitudes, mode of sonocation and time of treatment application of low frequency ultrasound on ML flux through Epiderm barrier. ANOVA one way analysis was used to evaluate the data and showed a significant difference between the control experiment and ultrasound treatments with $\mathrm{P}<0.001$. A post - hoc (LSD) test was carried out which showed that $15 \%$ amplitude and $20 \%$ amplitude for 5 minutes continuous sonication significantly improved the permeation of metronidazole through EpiDerm ${ }^{\mathrm{TM}}$ section. However neither power sonication at $10 \%$ amplitude nor physiotherapeutic US treatment for up to 20 minutes could enhance the permeation.

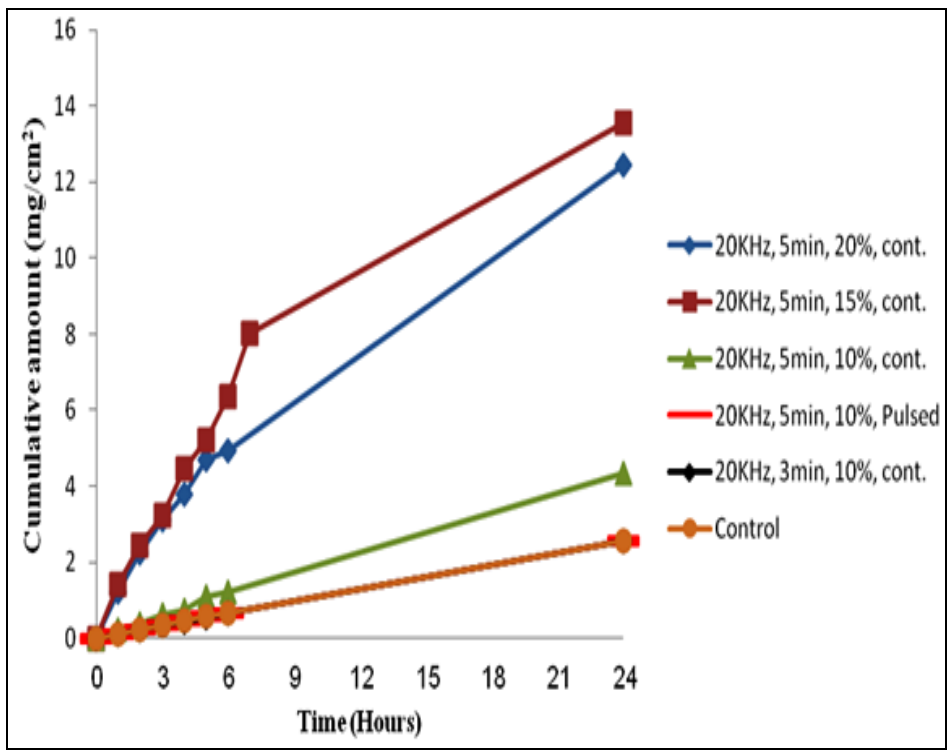

FIGURE 3: EFFECT OF LOW FREQUENCY US AT DIFFERENT AMPLITUDES OF $20 \mathrm{KHz}$ ON ML PERMEATION THROUGH EpiDerm $^{\text {TM }}$
DISCUSSION: Various hypotheses have been used to explain sonophoretic enhancement, the way of how US modifies and improve the permeation could not be answered by using a single mechanism because many different potentially modulating physical situation are generated simultaneously by ultrasonic wave, and the theoretical and experimental basis of the intricate mechanism is in its infancy ${ }^{7}$. Thermal effect and cavitation are the most significant mechanisms used to explain the effect of US 18, 19, 20, 21 . Generally; the mechanisms are divided into two main types: Thermal effects and non-thermal ${ }^{22,23,24}$.

Upon propagation of the ultrasonic waves through a medium, the pressure amplitude of the wave decreases with the distance and is attenuated due to either scattering or absorption of the ultrasonic beam by the medium. Absorption of the beam causes an increase in medium temperature as a result of converting ultrasonic energy into heat this will cause thermal effect (O'Brien 2007). The energy release from the collapsed transient cavitation bubbles participate in elevating the medium temperature; however this temperature increase is a function of the frequency, intensity and exposure time ${ }^{25}$.

For the purpose of minimizing the thermal contribution when studying the permeation improvement obtained across EpiDerm ${ }^{\mathrm{TM}}$ upon insonation, treating the EpiDerm $^{\mathrm{TM}}$ tissue with both a therapeutic probe and power $20 \mathrm{KHz}$ probe was performed in a beaker filled with PBS prior to fixing the tissue culture in a Franz cell, as some result showed negative results due to thermal effect which could promote the reverse diffusion of $\mathrm{ML}$ molecules to their vehicle gel rather than to pass through. Therefore it is thought that the effect of ultrasound on the permeation improvement of the investigated medicines might be due to the other ultrasonic mechanisms.

Power, therapeutic and high frequency ultrasound could enhance the permeation of drugs through different barriers and skin models investigated, in these studies the cavitation is the main mechanism suggested to explain the effect of US ${ }^{26-34}$. Acoustic cavitation is strongly dependent on the frequency of ultrasound in an inverse proportional relationship ${ }^{35}$. 
At therapeutic frequencies it may be only stable cavitation that takes place while at low frequencies both stable and transient cavitations occurs because at higher frequencies the time between negative and positive acoustic pressure becomes less than the time needed to develop a full gas bubble ${ }^{21}$. It was found that there is an intensity threshold below which no enhancement could be achieved. This threshold intensity increases with increasing frequency ${ }^{36}$.

Mitragotri et al., has suggested that the permeation enhancement under power in sonicating condition should be significantly better than that obtained utilizing therapeutic US because the rate of creating and collapsing of cavitation bubbles under the lower frequency US will be higher ${ }^{37}$. Indeed the permeation coefficients obtained by power frequency in this work were far higher than those obtained post therapeutic sonication.

Cavitation site of action could take place through the creation of gaseous bubbles and then the collapse of these bubbles after few ultrasonic cycles or immediately could take place significantly either inside the skin or outside the skin in the donor compartment utilizing the dissolved gases in the drug vehicle. Cavitation bubbles could be enhancing the permeation of drugs across stratum corneum utilizing different mechanisms produced by the two types of cavitation i.e. stable and transient bubbles.

Transient bubbles might collapse either in the bulk of donor compartment symmetrically, to produce a shock wave with amplitude of about 10 kbar, which can perturbate the structure of stratum corneum by the high pressure or near the skin surface without penetrating the $\mathrm{SC}$ resulting in stronger perturbation and finally by collapsing at the SC surface itself asymmetrically and thus produce high velocity liquid microjet which impinge on the stratum corneum surface in a velocity of about $100 \mathrm{~m} / \mathrm{s}^{38,39}$.

It was suggested that low frequency cavitation induce the penetration of water through the disordered stratum corneum lipids and thus forming aqueous channels through the stratum corneum which enhances the permeation of water soluble molecules 23
According to the results; we expect that EpiDerm ${ }^{\mathrm{TM}}$ tissue get defects following 3 minutes of power sonication at $10 \%$ amplitudes and defective regions increased upon increasing the sonication to 5 minutes. Shedding of the SC layer on EpiDerm ${ }^{\mathrm{TM}}$ is more when it was exposed to higher pressure amplitude 20\% (high intensity) see results. We concluded that there are no defects after therapeutic irradiating of the EpiDerm ${ }^{\text {TM }}$ at $1 \mathrm{MHz} 2 \mathrm{~W} / \mathrm{cm}^{2}$ for 20 minutes. So the enhancement obtained by therapeutic sonication could be to the cavitation of tiny bubbles (few micron in diameter) resulted from the stable cavitation, while the enhancement by power sonication is due to creating defects or aqueous channels across the stratum corneum resulted from transient cavitation which can produce bubbles with diameter up to $150 \mu \mathrm{m}$ each according to 40 .

Acoustic microstreaming which is the development of fluid high velocities in small scale eddying as a consequence of ultrasonic waves scatterings and reflections and to the oscillation and collapsing of the cavitation bubbles, create shear stresses which might play a role in improving the enhancement through disrupting the packed structure of the stratum corneum in the enhancement of drug penetration through the skin ${ }^{40}$.

This suggested mechanism may be of significant value in the work conducted using the EpiDerm ${ }^{\text {TM }}$ tissue as a model because this mechanism might explain the severity of the damage which was experienced when the work was performed with high pressure amplitudes (50\%), as the continuous creation of the aqueous channels within the tissue resulted in minimizing the binding strength between the cells of the epidermis of this model.

From previous work we might not assume that the sonophoretic enhancement of ultrasound is an effect of a single mechanism. In contrast it is the result of many mechanisms, which may vary according to the type of barrier, the ultrasound frequency, the pressure amplitude, the time and technique of exposing the barrier to the ultrasonic propagation. This might make the medical use of ultrasound for the co administration of potent drugs a hard goal to achieve ${ }^{41}$. 
CONCLUSION: Metronidazole permeation enhanced through skin using sonophoresis. The enhancment depends on US parameters such as frequency, intensity and duration of US applied. The use of 3D skin equivalent EpiDerm ${ }^{\mathrm{TM}}$ as a model for the human skin to investigate the influence of the physical penetration enhancement of ultrasound is possible, informative and offers the advantages of investigating the effect of ultrasound on viable human cells without the implications of the ethical constraints. Two types of US have been used: therapeutic and power ultrasound. The last was more profound on the permeation of Metronidazole across the EpiDerm.

Therapeutic ultrasound treatments had moderate, but not significant enhancement effects on the permeation of the model drugs, 10 minutes of continuous sonication at $1 \mathrm{MHz}$ frequency $2 \mathrm{~W} / \mathrm{cm}^{2}$ intensity has enhanced the permeation of Metronidazole by $168 \%$ and doubling the sonication time improved the permeation to $198 \%$. Power sonication at $20 \mathrm{KHz}$ and $10 \%$ amplitude for 5 minutes managed to improve the permeation of Metronidazole to $160 \%$. The pattern of better penetration enhancement for Metronidazole could still be observed upon increasing the pressure amplitude of the sonication to $15 \%$ and $20 \%$, where penetration of Metronidazole was enhanced by $473 \%$ and $443 \%$ respectively.

Cavitation could have played the most dominant role in the enhancement of permeation across the EpiDerm ${ }^{\mathrm{TM}}$. Oscillation and collapsing of the gaseous bubbles probably enhanced the permeation by minimizing the number of SC layers, creating aqueous channels which work as pathways for Metronidazole molecules. The mechanism of permeation enhancement is variable with the ultrasonic parameters, the length of ultrasonic treatment and the permeant physicochemical properties.

\section{REFERENCES:}

1. Prausntiz MR.: Microneedles for transdermal drug delivery. Adv Drug Deliv Rev 2004; 56, 581-587.

2. Lavon I, and Kost J.: Ultrasound and transdermal drug delivery. DDT 2004; 9: 670-676.

3. Meshali M., Abdel-Aleem H., Saker F., Nazzal S., and El-Malah Y.: Effect of gel composition and phonophoresis on the transdermal delivery of ibuprofen: In vitro and in vivo evaluation. Pharmaceutical Development and technology 2011; 16(2), 93-101.
4. William AC, Barry BW: Penetration enhancers. Adv Drug Deliv Rev 2004; 56, 603-618.

5. Prausnitz MR and Langer R.: Transdermal drug delivery. Nat Biotechnol 2008; 26, 1261-1268.

6. Polat, B.E., Hart, D., Langer, R., Blankschtein, D.: Ultrasoundmediated transdermal drug delivery: mechanisms, scope, and emerging trends. J. control. Release 2011; 152, 330-348.

7. Rao R., and Nanda S.: Sonophoresis: recent advancements and future trends. Journal of Pharmacy and Pharmacology 2009; 61, 689-70.

8. Ogura M, Paliwal S, Mitragotri S.: Low-ferquency sonophoresis: Current status and future prospects. Adv Drug Deliv Rev 2008; 60, 1218-1223.

9. Fiorillo AS., grimaldi D., Paolino D., and Pullano S.A.: Low frequency ultrasound in medicine: An In Vivo Evaluation. IEEE transactions on instrumentation and measurement 2012; 6 (6).

10. Park D., Ryu H., Kim H.S., Seo J.: Sonophoresis using ultrasound contrast agent for transdermal drug delivery: an in Vivo experimental study, Ultrasound in Med. \& Biol.2012; 38 (4), 642-650.

11. Herwadkar A., Sachdeva V., Taylor L.F., Silver H., and Banga A.K.: Low Frequency Sonophoresis Mediated Transdermal And Intradermal Delivery Of Ketoprofen. International Journal of Pharmaceutics 2012; 423, 289-296.

12. Aldwaikate M.: The Effect of Ultrasound on Transdermal Drug Delivery, PhD thesis 2007.

13. Schnetz E. and Fartasch M.: Microdialysis for the evaluation of penetration through the human skin barrier - a promising tool for future research?' The European journal of pharmaceuticals 2001; 12 (3), 165-174.

14. Malan S., Chetty D. and Plessis J.: Physicochemical properties of drugs and membrane permeability. South African journal of science 2002; 98 (7-8), 385-391.

15. Maruani A., Boucaud A., Perrodeau E., Gender B., and Machet L.: Low-frequency ultrasound sonophoresis to increase the efficiency of tropical steroids: a pilot randomized study of humans. International journal of pharmaceutics 2011; 395, 8490.

16. McElnay J., Kennedy T., and Harland R.: The influence of ultrasound on the percutaneous absorption of fluocinolone acetonid. International Journal of Pharmaceutics 1987; 40 (12), $105-110$.

17. [17] Mitragotri S., Blankschtein D. and Langer R.: An explanation for the variation of the sonophoretic transdermal transport enhancement from drug to drug' J. of Pharmaceutical sciences 1997; 86 (10), 1190 - 1192.

18. Jambrak Anet Rezek, Lelas Vesna, Herceg Zoran, Badanjak Marija, Batur Verica, and Muza Mislav: Advantages and disadvantages of high power ultrasound application in the dairy industry. Mljekarstvo 2009; 59 (4), 267-281.

19. Nanzai Ben, Okitsu Kenji, Takenaka Norimichi, and Bandow Hiroshi: Effects of initial concentration of LASs on the rates of sonochemical degradation and cavitation efficiency. Research on Chemical Intermediates 2009; 35 (8-9), 841-849.

20. Jang Hyun Joo, Lee Jae-Young, Lee Don-Haeng, Kim Won-Hong, and Hwang, Joo Ha: Current and Future Clinical Applications of High-Intensity Focused Ultrasound (HIFU) for Pancreatic Cancer. Gut and Liver 2010; 4 (1), S57-S61.

21. Ahmadi F., McLoughlin I. V., Chauhan S., and ter-Harr G.: Bioeffects and safety of low-intensity, low frequency ultrasonic exposure, Progress in biophysics and Molecular Biology 2012; $108,119-138$. 
22. ter Haar G. and Daniels S.: Evidence of ultrasonically induced cavitation in vivo. Physics in medicine and biology 1981; 26 (6), $1145-1149$.

23. Mitragotri S.: Modeling skin permeability to hydrophilic and hydrophobic solutes based on four permeation pathways' J. of Controlled release 2002; 86 (1), 69-92.

24. Merino G., Kalia Y. and Guy R.: Ultrasound enhanced transdermal transport. J. of pharmaceutical sciences 2003; 92 (6), 1125-1137.

25. Ebrahimi S., Abbasnia K., Motealleh A., Kooroshfard N., Kamali F., and Ghaffarinezhad F.: Effect of lidocaine phonophoresis on sensory blockade: pulsed or continuous mode of therapeutic ultrasound?. Physiotherapy 2012; 57-63.

26. Bomannan D., Menon G., Okuyama H., Elias P. and Guy R.: Sonophoresis II. Examination of the mechanisms of ultrasound enhanced transdermal drug delivery. Pharmaceutical research 1992; 9 (8), 1043-1051.

27. Mitragotri S., Edwards D. Blankschtein D. and Langer R.: A mechanistic study of ultrasonically enhanced transdermal drug delivery. Journal of pharmaceutical sciences 1995; 84 (6), 697706.

28. Fang J., Fang C., Sung K., and Chen H.: Effect of law frequency ultrasound on the in-vitro percutaneous absorption of clobetasol 17 -propionate. International J. of pharmaceutics 1999; 191 (1), 33-42.

29. Frenkel V., Kimmel E. and Yoni I.: Ultrasound facilitated transport of silver chloride particles in fish skin. J. of controlled release 2000; 68 (2), 251-261.

30. Boucaud A., Machet L., Arbeille B., Machet M. Sournac M., Mavon A. Patat F. and Vaillant L.: In vivo study of low frequency ultrasound -enhanced transdermal transport of fentanyl and caffeine across human and hairless rat skin. International J. of pharmaceutics 2001; 228 (1-2), 69-77 and Boucaud A., Montharu J., Machet L., Arbeille B., Machet M. Sournac M., Patat F. and Vaillant L.: Clinical, histological and electron microscopy study of skin exposed to low frequency ultrasound. The anatomical record 2001; 264 (1), 114-119.
31. Boucaud A., Barrigue M., Machet L., Vaillant L. and Patat F.: Effect of sonication parameters on transdermal delivery of insulin to hairless rat. Journal of controlled release 2002; 81 (12), 113-119.

32. Weimann L. and Wu J.: Transdermal delivery of Poly-L-Lysine by sonomacroporation. Ultrasound in medicine and biology 2002; 28 (9), 1173-1180.

33. Terahara T., Mitragotri S., and Langer R.: Porous resins as a cavitation enhancer for low frequency sonophoresis. Journal of Pharmaceutical sciences 2002; 91 (3), 753-759.

34. Merino G., Kalia Y., Charro M., Potts R. and Guy R.: Frequency and thermal effects on the enhancement of transdermal transport by sonophoresis. Journal of Controlled Release 2003; 88 (1), 85-94.

35. Matusomoto Y., Allen J., Yoshizawa S., Ikeda T. and Kaneko Y.: Medical ultrasound with microbubbles. Experimental thermal and fluid science $2005 ; 29$ (3), $255-265$.

36. Tezel A., Sens A., Tuchschere J. and Mitragotri S.: Frequency dependence of sonophoresis. Pharmaceutical research 2001;18 (12), 1694-1700.

37. Mitragotri S., Blankschtein D. and Langer R.: Transdermal drug delivery using low frequency ultrasound. Pharmaceutical research 1995; 13 (3), 411-420.

38. Tang H., Wang C., Blankschtein D. and Langer R.: An investigation of the role of cavitation in low frequency ultrasound mediated transdermal drug transport. Pharmaceutical research 2002; 19 (8), 1160-1169.

39. Tezel A. and Mitragotri S.: Interactions of inertial cavitation bubbles with stratum corneum lipid bilayers during low frequency sonophoresis. Biophysical Journal 2003; 85 (6), 30523512.

40. Simonin J.P.: On The Mechanisms Of In-Vitro And In-Vivo Phonophoresis. Journal of Controlled Release 1995; 33 (1), 125141.

41. Smith N. B.: Prespectives on transdermal ultrasound mediated drug delivery. International journal of Nanomedicine 2007; 2 (4), 585-594.

How to cite this article:

Aldwaikat M, Alarjah M, Willis J, Mason T: Sonophoresis Effect on the Permeation of Metronidazole Using 3D Skin Equivalent. Int J Pharm Sci Res. 2013; 4(1); 205-214. 